\title{
INTERVENÇÕES COMPORTAMENTAIS PARA O TRANSTORNO DE ESCORIAÇÃO: REVISÃO DE ARTIGOS PUBLICADOS EM PERIÓDICOS DE SAÚDE
}

\author{
BEHAVIORAL INTERVENTIONS FOR EXCORIATION DISORDER: REVIEW OF PAPERS PUBLISHED IN \\ HEALTH JOURNALS
}

\author{
MARISA RICHARTZ
}

MÁrcia Cristina CASERTA Gon

UNIVERSIDADE ESTADUAL DE LONDRINA, BRASIL

ROBSON ZAZULA

UNIVERSIDADE FEDERAL dA INTEGRAÇÃo LATINO-AMERICANA, BRASIL

\begin{abstract}
RESUMO
O transtorno de escoriação é definido por um excesso de comportamentos de arranhar, ferir ou esfolar a pele. Os comportamentos não estão relacionados a problemas no neurodesenvolvimento e provocam sofrimento emocional e prejuízos na vida social de quem os apresenta. Além de ser considerado um problema dermatológico, por afetar diretamente a pele e seus anexos, esse transtorno também apresenta características psiquiátricas, recebendo atenção de pesquisadores da área. Conduziu-se uma revisão bibliográfica em periódicos da saúde com os objetivos de identificar pesquisas aplicadas ou relatos de casos realizados com indivíduos com desenvolvimento típico que apresentassem escoriação, descrever as intervenções comportamentais empregadas e relatar os métodos e os resultados dos procedimentos. Uma pesquisa eletrônica foi realizada nas bases de dados PsycINFO, Web of Science, PubMed/MEDLINE e BVS com os seguintes descritores: 'escoriação', 'neurotic excoriation' ou psychogenic excoriation'. Esses foram combinados com 'behavioral treatment'. Dezoito artigos foram recuperados, catalogados e analisados qualitativamente a partir dos objetivos, métodos empregados e resultados das intervenções. Constatou-se que a maioria das pesquisas foi conduzida com adultos e adotaram-se estratégias e métodos da terapia de reversão de hábito e da terapia de aceitação e compromisso. Observou-se que essas intervenções foram efetivas para garantir redução da frequência da escoriação, porém com dificuldade na manutenção dos resultados na avaliação de seguimento. Sugerem-se futuras pesquisas com maior diversidade de variáveis e de participantes, bem como a avaliação funcional do comportamento de escoriação.
\end{abstract}

Palavras-chave: transtorno de escoriação, escoriação psicogênica, skin picking, terapia de reversão de hábito, intervenção comportamental

Excoriation disorder is defined by excessive and recurrent behavior of scratching or injuring the skin. It is not related to neurodevelopment problems, it causes emotional suffering and it negatively affects the social life of those who present the condition. Besides being considered a dermatological problem, as it directly involves the skin and its attachments, the disorder is also a psychiatric condition that has been receiving attention from mental health researchers. A bibliographic review was conducted in health journals in order to identify applied studies and case reports with typically developing individuals that excoriate the skin, to present behavioral interventions employed as treatment, and to report the method and results of the adopted procedures. It was carried out an electronic search at PsycINFO, Web of Science, PubMed / MEDLINE and BVS databases using 'excoriation', 'neurotic excoriation' or 'psychogenic excoriation', combined with 'behavioral treatment', as descriptors. Eighteen articles were retrieved, cataloged and qualitatively analyzed based on their objectives, methods and results. It was found that most of the studies involved adults and used habit reversal therapy and acceptance and commitment therapy. It was observed that these interventions were effective in reducing excoriation frequency, however follow-up evaluation indicated difficulty in maintaining the effects. It is suggested future research on excoriation disorder with more diversity regarding variables and patients. Functional analysis of excoriation behavior is also recommended. intervention.

Key words: excoriation disorder, psychogenic excoriation, skin picking, habit reversal therapy, behavioral

Agradecemos à Coordenação de Aperfeiçoamento de Pessoal de Nível Superior (CAPES) pelo apoio financeiro durante a realização da pesquisa. Correspondência referente a este artigo devem ser enviadas a marichartz@gmail.com 
O transtorno de escoriação é caracterizado por comportamentos repetitivos e compulsivos de picar, escoriar, coçar, arranhar ou furar determinadas regiões da própria pele, que resultam em danos no tecido da pele (Grant \& Odlaug, 2009; Odlaug \& Grant, 2010). O indivíduo é parcialmente consciente da emissão desses comportamentos e o admite, se lhe for perguntado (Harth, Taube, \& Gieler, 2010). É um comportamento que pode ter como consequências o surgimento de cicatrizes e deformações na pele (Wetterneck, Teng, \& Stanley, 2010) e acomete, em média, $3 \%$ da população adulta, especialmente mulheres (Flessner, Busch, Heideman, \& Woods, 2008). Por esta razão é considerado um problema dermatológico, compondo a categoria denominada dermatite paraartefacta e dermatite factícia (Harth et al., 2010; Shah \& Fried, 2006).

As doenças da pele factícias são caracterizadas por lesões que não são explicadas por condição dermatológica orgânica e são produzidas pelo próprio indivíduo (Shah \& Fried, 2006). Movimentos mecânicos (e.g., friccionar, esfregar, morder, cutucar a pele), uso de substâncias tóxicas (e.g., ácidos), indução de infecção por não deixar ferimentos cicatrizarem e uso de medicações que afetam a pele (e.g., injeções de heparina e insulina) são exemplos de comportamentos que produzem dermatites de autolesão. Um tipo especial dessa condição é a escoriação da acne, que ocorre quando o comportamento de escoriação é realizado na face, nos locais de lesão da acne (Harth et al., 2010).

Além de ser considerado um problema dermatológico, por afetar diretamente a pele e seus anexos, o transtorno de escoriação também apresenta características de transtornos psiquiátricos, recebendo atenção de diversos pesquisadores (e.g., Deckerbach, Wilhelm, Keuthen, Baer, \& Jenike, 2002). O Manual Diagnóstico e Estatístico de Transtornos Mentais, até a quarta versão revisada (DSM IV-TR; APA, 2000) não apresentava uma classificação específica para essa condição, a qual compartilhava características com o transtorno de controle de impulso (Harth et al., 2010) e, em alguns casos, era comparado à tricotilomania (Flessner et al., 2008). Na quinta versão do Manual Diagnóstico e Estatístico de Transtornos Mentais (DSM V; APA, 2013), o transtorno de escoriação foi classificado e definido pelo excesso de comportamento de beliscar a pele e déficit de respostas em controlar o impulso para tal ação, não estando relacionado a delírios, ao transtorno dismórfico corporal, tiques ou movimentos estereotipados ligados aos transtornos do neurodesenvolvimento. Como consequência dos excesso e déficit comportamentais, observa-se um sofrimento emocional clinicamente relevante para o indivíduo, bem como prejuízos em nível social e profissional em outras áreas da vida. Esses prejuízos podem estar relacionados ao tempo que o indivíduo gasta com o comportamento de escoriar as lesões, causando danos no tecido cutâneo e, consequentemente, levando a uma aparência não estética da pele (APA, 2013).

Portanto, o transtorno de escoriação pode ser caracterizado como uma dificuldade de controlar comportamentos de escoriar a pele que, como consequência, produz alterações cutâneas que se relacionam com prejuízos para o corpo, discriminação e dificuldades na interação social (Flessner et al., 2008). Uma alteração na pele do indivíduo, ao ser evidente e apresentar-se como uma distorção na conformação corporal, comumente gera em outras pessoas comportamentos de preconceito e pode dificultar as interações pessoais (Gon, Rocha, \& A. Gon, 2005; Gon, A. Gon, \& Zazula, 2013). Por sua vez, de acordo com Gupta e A. K Gupta (1996), provocar lesões na própria pele tem relação com sentimentos de inadequação social, insegurança, sensação de vazio e solidão.

Diante das graves consequências do transtorno de escoriação para o indivíduo que o apresenta, o estabelecimento de tratamentos eficazes é primordial. Lochner, Roos e Stein (2017) realizaram uma revisão sistemática de literatura com o objetivo de atualizar o leitor sobre formas de tratamento. Por meio dessa revisão, foram identificados tratamentos não farmacológicos (e.g., Terapia Cognitivo Comportamental e Terapia de Reversão de Hábito - TRH), farmacológicos (e.g., inibidores seletivos de receptação de serotonina, lamotrigina, agentes glutaminérgicos, naltrexona como antagonista de opióide, inisitol e estratégias de potencialização com antipsicóticos típicos e atípicos) e intervenções alternativas (e.g., ioga, exercícios aeróbicos e hipnose). Os autores não encontraram estudos que combinavam tratamento psicofarmacológico e psicológico, resultado que corrobora os de Selles et al., (2016). Em uma revisão de literatura sistemática com meta análise, que objetivou avaliar a eficácia de tratamentos, Schumer, Bartley e Bloch (2016) concluíram que: (a) as intervenções comportamentais (e.g., terapia cognitiva e comportamental e TRH) foram benéficas nos estudos com grupo controle; (b) os tratamentos farmacológicos com inibidores seletivos de receptação de serotonina ou lamotrigina não mostraram resultados significativos em estudos realizados com grupo controle; (c) as intervenções comportamentais e tratamentos farmacológicos se mostraram eficazes em estudos que não utilizaram grupo controle. Selles et al. (2016), ao analisar estudos sobre tratamento, encontraram diferentes formas de aplicação dos procedimentos da terapia cognitivo comportamental e concluíram que as intervenções comportamentais são recomendadas como forma de tratamento inicial, especialmente por apresentarem mais evidências científicas de efetividade, quando comparadas ao tratamento psicofarmacológico. No entanto, os autores desses estudos não especificaram os componentes das intervenções comportamentais e excluíram estudos de caso.

Diante disso, conduziu-se uma revisão bibliográfica em periódicos da saúde com o objetivo de identificar pesquisas aplicadas ou relatos casos realizados com indivíduos com desenvolvimento típico que praticassem o comportamento de escoriação, apresentar o método e os principais componentes das intervenções comportamentais empregadas e relatar os resultados desses procedimentos. Portanto, o presente estudo diferencia-se dos de Lochner et al. (2017), Selles et al. 
(2016) e Schumer et al. (2016) por incluir estudos de caso e apresentar e destacar os principais componentes das intervenções comportamentais.

\section{MÉTODO}

Foi realizada uma revisão bibliográfica em três etapas. A primeira etapa consistiu na realização de uma pesquisa eletrônica em fevereiro de 2018, nas bases de dados PsycINFO, Web of Science, PubMed/MEDLINE e BVS. Foram utilizados os seguintes descritores, da seguinte forma: 'escoriação', 'neurotic excoriation' ou psycogenic excoriation' combinados com 'behaviorial treatment'. Na base de dados PsycINFO as palavras foram buscadas, apenas, como palavra-chave; na base Web of Science, no título ou resumo; e na base Web of Science e na base BVS, no título, resumo e assunto. Não houve delimitação quanto ao período de publicação dos artigos.

$\mathrm{Na}$ segunda etapa foi realizada a seleção dos artigos para o presente estudo. Esses foram selecionados a partir da leitura do título e do resumo. Foram selecionados os artigos que descreviam(a) estudos que incluíam tratamentos não farmacológicos para o transtorno de escoriação e (b) intervenções realizadas com participantes com desenvolvimento típico. Foram excluídos artigos que descreviam: (a) tratamentos psicofarmacológicos, (b) intervenções direcionadas a participantes com desenvolvimento atípico que apresentavam síndrome do espectro autista ou transtorno global do desenvolvimento ou transtornos do neurodesenvolvimento; (c) descrição ou apresentação de protocolos de intervenção que não se baseavam na terapia comportamental ou na terapia cognitivo-comportamental (e.g., estudos que descreviam hipnose como forma de tratamento), (d) caracterização epidemiológica da população-alvo; (e) artigos de revisão de literatura, (f) resenhas ou cartas ao editor das revistas e (g) artigos que não apresentam resumo disponível nas bases de dados consultadas (e.g., artigos com publicação na década de 1940). As referências dos artigos levantados na pesquisa eletrônica foram examinadas e artigos que estivessem em conformidade com os critérios de inclusão e exclusão foram selecionados.

Após a seleção foi realizada a terceira etapa que consistiu na leitura dos artigos na íntegra e análise qualitativa, a qual focalizou as seguintes categorias: (a) método, envolvendo a descrição e análise dos objetivos, perfil e quantidade dos participantes, delineamento de pesquisa adotado e indicadores analisados, (b) intervenções, por meio do número de sessões realizadas e procedimentos adotados e (c) resultados das intervenções.

\section{RESULTADOS}

Foram selecionados 18 artigos para análise, sendo 13 resultantes das buscas nas bases de dados (ver Tabela 1) e cinco encontrados a partir da seção de referências dos artigos analisados. Dois artigos selecionados na Fase 2, por meio da base das pesquisas nas bases de dados (Fruensgaard, 1991b; Keuthen et al., 2015) não foram encontrados pelos autores para leitura na íntegra e por isso, foram excluídos do estudo. Nenhum artigo nacional foi selecionado na busca bibliográfica.

Tabela 1.

Dados da Busca Realizada nas Bases de Dados.

\begin{tabular}{lcc} 
Base de Dados & $\begin{array}{c}\text { Artigos } \\
\text { encontrados }\end{array}$ & $\begin{array}{c}\text { Artigos } \\
\text { selecionados }\end{array}$ \\
\hline PsycINFO & 171 & 10 \\
Web of Science & 148 & 2 \\
PubMED & 4 & $2^{\mathrm{a}}$ \\
BVS & 0 & 0
\end{tabular}

${ }^{a}$ Um dos artigos (Deckersbach et al., 2002) selecionados por essa base dados foi também selecionado no PsycINFO.

Os artigos selecionados foram publicados no período de 1968 a 2017, sendo que 15 deles foram publicados após 2000 (Capriotti, Ely, Snorrason, \& Woods, 2015; Deckersbach, Wilhelm, Keuthen, Baer, \& Jenike, 2002; Flessner, Busch, Heidman, \& Woods, 2008; Flessner, Moutoun-Odum, Stocker, \& Keuthen, 2007; Grillo, Long, \& D. Long, 2007; Ibiloglu et al., 2016; Jagger \& Sterner 2016; Long, R. Long, Grillo, \& Marshman, 2006; Martinson, Nangle, Boulard, \& Sigmon, 2011; Schuck, Keijsers, \& Rinck, 2011; Moritz, Fricke, Treszl, \& Wittekind, 2012; O’Connor, Lavoie, Desaulniers, \& Audet, 2018; Teng, Woods, \& Twohig, 2006; Twohig, Hayes, \& Masuda, 2006; Twohig \& Woods, 2001). Um artigo foi publicado no início da década de 90 (Fruensgaard, 1991a) e outro no início da década de 80 (Rosenbaum e Ayllon, 1981). Apenas um foi publicado anteriormente aos anos 70 (Pearlstein \& Orentreich, 1968).

\section{Análise qualitativa do método Descrição e Análise dos Objetivos.}

Três estudos avaliaram a efetividade da TRH (Rosenbaum \& Ayllon, 1981; Teng et al., 2006; Twohig \& Woods, 2001), três objetivaram descrever procedimentos baseados na terapia de aceitação e compromisso (ACT; Capriotti et al., 2015; Flessner et al., 2008; Twohig et al., 2006) e dois estudos realizaram procedimentos de terapia cognitivo-comportamental para pacientes com escoriação (Deckersbach et al., 2002; Schuck, Keijers, \& Rinck, 2011). Além disso, uma publicação avaliou a eficácia de um tratamento combinado - cognitivo e psicofisiológico (CoPs) na redução dos sintomas de comportamentos repetitivos (O'Connor et al., 2018) e outra descreveu uma proposta de intervenção em plataforma online como estratégia de tratamento do transtorno de escoriação (Flessner et al., 2007). Um segundo estudo comparou os efeitos das intervenções de TRH e de uma técnica chamada decoupling, quando aplicadas pelo próprio indivíduo a partir de informações obtidas online (Moritz et al., 2012). Dois estudos objetivaram apresentar programas estruturados de intervenção para doenças da pele em geral, dentre elas o transtorno de escoriação (Grillo et al., 2007; 
Long et al., 2006). Fruensgaard (1991a) esboçou um planejamento de estratégias psicoterapêuticas para o tratamento do transtorno de escoriação a partir de informações coletadas com pacientes de um serviço de dermatologia. Por fim, quatro estudos apresentaram relatos de casos com descrições de tratamentos (Ibiloglu et al., 2016; Jagger \& Sterner, 2016; Martinson et al., 2011; Pealrstrein \& Orenterich, 1968).

\section{Perfil e Quantidade dos Participantes}

Em relação ao perfil dos participantes, em 17 estudos esses eram adultos, com idade superior a 18 anos e com desenvolvimento típico. Quanto aos critérios de inclusão e exclusão de participantes utilizados nos estudos, pode-se observar diversidade entre eles, tais como no estudo de Flessner et al. (2008), que excluiu participantes em condições psiquiátricas (e.g., transtorno psicótico, abuso de substância e outras condições que impediriam a possibilidade de locomoção para as sessões de tratamento) e o estudo de Twohig et al. (2006), que foi conduzido com participantes que não tomavam medicamentos psicotrópicos no período de pesquisa. O'Connor et al. (2018) excluíram do estudo todos os participantes com problemas neurológicos ou psicológicos maiores ou que realizaram alterações de medicação durante o período da pesquisa. Uma pesquisa selecionou participantes com doenças da pele em geral que se relacionavam a prurido (i.e., eczema, psoríase, prurido nodular e escoriação; Grillo et al., 2007) e outra ofereceu intevenção para escoriação juntamente com tratamento para tricotilomania o onicofagia (O'Connor et al., 2018).

O número de participantes selecionados variou conforme o método e delineamentos propostos. Nos dez estudos em que foram realizadas análises individuais, o número de participantes variou entre um e seis (Capriotti et. al, 2015; Deckersbach et al., 2002; Flessner et al., 2008; Ibiloglu et al., 2016; Jagger \& Sterner, 2016; Martinson et al., 2011; Pearlstein \& Orentrich, 1968; Rosenbaum \& Ayllon, 1981; Twohig \& Woods, 2001; Twohig et al., 2006). Quatro utilizaram delineamento de grupo, sendo um com 19 participantes (Teng et al., 2006), um com 34 participantes (17 no grupo experimental e 17 no grupo controle; Schuck et al., 2011), um com 70 participantes (35 em cada grupo: (Moritz et.al, 2012) e outro com 47 (O’Conner et al., 2011). Um estudo realizou análises estatísticas em um único grupo de 372 participantes (Flessner et al., 2007).

\section{Delineamento de Pesquisa Adotado e Indicadores Analisados.}

Em relação ao método, seis estudos utilizaram delineamento de sujeito único (Capriotti et al, 2015; Deckersbach et al., 2002; Rosenbaum e Ayllon, 1981; Twohig et al., 2001, 2006; Martinson et al., 2011), seis de grupo (Flessner et al., 2007; Grillo et al.; 2007; O'Connor et al., 2018; Shuck et al., 2011; Teng et al., 2006; Moritz et. al., 2012) e um de linha de base múltipla e linha de base com reversão (Flessnner et al., 2008). Cinco estudos não apresentaram dados empíricos sobre a intervenção e por isso Tabela 2. foram classificados como relatos de caso (Fruensgaard, 1991a; Ibiloglu et al., 2016; Jagger \& Sterner, 2016; Long et al., 2006; Pearlstein \& Orentreich, 1968), conforme Tabela 4.

A mensuração do comportamento-alvo foi feita indiretamente por meio de estratégias de relato e de instrumentos padronizados. Dentre os indicadores indiretos destacam-se o autorregistro (Capriotti et al., 2015; Flessner et al., 2007, 2008; Martinson et al., 2011; Moritz et al., 2012; O’Connor et al., 2018; Teng et al., 2006; Twohig \& Woods; 2001; Twohig et al., 2006), análise de fotografias das lesões antes e após o tratamento (Teng et al., 2006; Twohig \& Woods, 2001; Twohig et al., 2006; Pearlstein \& Orentriech, 1968) e escalas e testes

\section{Análise Qualitativa das Intervenções \\ Número de Sessões Realizadas.}

O número de sessões nos estudos variou de uma a 40 e a duração variou entre 30 minutos e uma hora. Teng et al. (2007) e Twohig e Woods (2001) realizaram três sessões com periodicidade semanal, sendo a primeira sessão com duração de uma hora e as demais com duração de trinta minutos. Schuck et al. (2011) conduziram quatro sessões, com periodicidade semanal e duração de 45 minutos cada uma. Os autores que utilizaram estratégias baseadas na ACT realizaram um número maior de sessões, sendo que Twohig et al. (2006) conduziram a intervenção em oito sessões, com periodicidade semanal e Flessner et al. (2008) conduziram a intervenção em dez sessões, com 50 minutos de duração e periodicidade semanal.

Deckersbach et al. (2002) realizaram, respectivamente, três estudos de caso com quatro, seis e sete sessões, conforme análise funcional prévia com os participantes. Capriotti et al. (2015) apresentaram quatro estudos de caso, os quais tiveram dez sessões com duração de uma hora. Um dos participantes desse estudo realizou sessões adicionais, totalizando 25 sessões. O'Connor et al. (2018) também realizaram dez sessões. Rosenbaum e Ayllon (1981) realizaram estudos de caso com quatro pacientes e uma única sessão.

O programa elaborado por Flessner et al. (2007), com procedimento autoaplicável, foi dividido em três módulos (i.e., avaliação, intervenção e manutenção) e não houve definição de qual era a quantidade mínima ou máxima de sessões para cada um dos participantes. Moritz et al. (2012) também propuseram procedimentos autoaplicáveis via internet, sendo que o participante recebia instruções a partir de um material disponibilizado por email, sem informações sobre sessões. A proposta de Long et al. (2006), apresentou apenas um estudo de relato, sem dados empíricos e previa a realização de três sessões com os pacientes. Outros dois estudos apenas realizaram indicações de intervenções e não descreveram número ou duração de sessões (Fruensgaard, 1991a; Jagger \& Sterner, 2016). Ibiloglu et al. (2016) em um relato de um caso realizou consultas a cada duas semanas. A intervenção relatada no estudo de caso de Martinson et al. (2011) foi realizada durante 11 meses, com sessões com duração de 50 minutos e periodicidade semanal, totalizando 40 sessões. 


\begin{tabular}{|c|c|c|c|c|c|}
\hline \multirow{2}{*}{ Artigo } & \multicolumn{5}{|c|}{ Participantes } \\
\hline & Número & Gênero & Idade (anos) & Doença de pele & Desenvolvimento \\
\hline \multirow{2}{*}{$\begin{array}{l}\text { Deckersbach et al. } \\
(2002)\end{array}$} & \multirow[t]{2}{*}{3} & Masc. (1) & \multirow[t]{2}{*}{$57,26,51$} & \multirow[t]{2}{*}{ Escoriação } & \multirow[t]{2}{*}{ Típico } \\
\hline & & Fem. (2) & & & \\
\hline Flessner et al. (2007) & 372 & $\begin{array}{l}\text { Masc. (62) Fem. } \\
\text { (311) }\end{array}$ & $\begin{array}{c}\text { Entre } 30 \mathrm{e} \\
\quad 70\end{array}$ & $\begin{array}{l}\text { Escoriação e doenças } \\
\text { de pele }\end{array}$ & Sem descrição \\
\hline \multirow[t]{2}{*}{ Flessner et al. (2008) } & \multirow[t]{2}{*}{6} & \multirow[t]{2}{*}{-} & \multirow[t]{2}{*}{$>18$} & \multirow{2}{*}{$\begin{array}{l}\text { Escoriação e } \\
\text { tricotilomania }\end{array}$} & Típico \\
\hline & & & & & $(\mathrm{QI}<79)$ \\
\hline \multirow[t]{2}{*}{ Grillo et al. (2007) } & \multirow[t]{2}{*}{17} & Masc. (10) & \multirow{2}{*}{$\begin{array}{c}\text { Entre } 30 \mathrm{a} \\
\quad 70\end{array}$} & \multirow{2}{*}{$\begin{array}{l}\text { Doenças da pele em } \\
\text { geral }\end{array}$} & \multirow[t]{2}{*}{ Sem descrição } \\
\hline & & Fem. (7) & & & \\
\hline Long et al. (2006) & 0 & - & - & $\begin{array}{l}\text { Doenças da pele que } \\
\text { geram prurido }\end{array}$ & - \\
\hline \multirow[t]{2}{*}{ Teng et al. (2006) } & \multirow[t]{2}{*}{19} & \multirow[t]{2}{*}{ Fem. } & \multirow[t]{2}{*}{$>18$} & \multirow[t]{2}{*}{ Escoriação } & Típico \\
\hline & & & & & $(\mathrm{QI}<79)$ \\
\hline $\begin{array}{l}\text { Rosenbaum e Ayllon } \\
\text { (1981) }\end{array}$ & 4 & Fem & $\begin{array}{c}16,22,29 \mathrm{e} \\
34\end{array}$ & Escoriação & Típico \\
\hline \multirow[t]{2}{*}{ Schuck et al. (2011) } & \multirow{2}{*}{$\begin{array}{c}34(\mathrm{GC}=17 ; \\
\mathrm{GI}=17)\end{array}$} & Fem. (30) & \multirow{2}{*}{$\begin{array}{c}\text { Entre } 18 \mathrm{e} \\
38\end{array}$} & \multirow[t]{2}{*}{ Escoriação } & \multirow[t]{2}{*}{ Típico } \\
\hline & & Masc. (4) & & & \\
\hline $\begin{array}{l}\text { Twohig e Woods } \\
\text { (2001) }\end{array}$ & 2 & Masc. & 20 e 22 & Escoriação & Típico \\
\hline Twohig et al. (2006) & $6(5)^{a}$ & Fem. (5) & $\begin{array}{c}\text { Entre } 19 \mathrm{e} \\
24\end{array}$ & Escoriação & Típico \\
\hline \multirow{2}{*}{$\begin{array}{l}\text { Capriotti et al. } \\
\text { (2015) }\end{array}$} & \multirow[t]{2}{*}{4} & Fem (3) & \multirow[t]{2}{*}{$26,34,7$ e 32} & \multirow[t]{2}{*}{ Escoriação } & \multirow[t]{2}{*}{ Sem descrição } \\
\hline & & Masc. (1) & & & \\
\hline $\begin{array}{l}\text { Martinson et al. } \\
\text { (2011) }\end{array}$ & 1 & Fem & 55 & Escoriação & Sem descrição \\
\hline $\begin{array}{l}\text { Jagger e Stanner } \\
(2016)\end{array}$ & 1 & Fem & 33 & Escoriação & Sem descrição \\
\hline Ibiloglu et al. (2016) & 1 & Fem & 26 & Escoriação & Sem descrição \\
\hline Moritz et al. (2011) & 70 & $88 \%$ Fem GTRH & 28,37 & Escoriação & Sem descrição \\
\hline & $\left(\mathrm{GTRH}=5^{\mathrm{b}}\right.$ & 97\% Fem GDC & $(\mathrm{G} \perp \mathrm{RH})$ & & \\
\hline & $\left.\mathrm{GDC}=35^{\mathrm{c}}\right)$ & & $\begin{array}{l}29,54 \\
\mathrm{GT}=\mathrm{DC}\end{array}$ & & \\
\hline $\begin{array}{l}\text { Pearlstein e } \\
\text { Orentrich(1968) }\end{array}$ & 1 & Fem. & 45 & Escoriação & Sem descrição \\
\hline Fruensgaard (1991) & $\begin{aligned} 63(\mathrm{GI} & =25^{\mathrm{d}} ; \mathrm{GII} \\
& \left.=38^{\mathrm{e}}\right)\end{aligned}$ & $87 \%$ Fem & $\begin{array}{l}\text { Entre } 14 \mathrm{e} \\
77(\mathrm{M}=44)\end{array}$ & Escoriação & $\begin{array}{l}\text { Sem descrição } \\
\text { detalhada }\end{array}$ \\
\hline $\begin{array}{l}\text { O'Connor et al. } \\
(2018)\end{array}$ & $74^{\mathrm{f}}$ & $86,5 \%$ Fem & $\mathrm{M}=38,77$ & Comp. repetitivos & $\begin{array}{c}\text { Típico } \\
(\mathrm{QI}<79)\end{array}$ \\
\hline
\end{tabular}

Nota: GC= Grupo controle; GI = Grupo intervenção; CTRH = Grupo de Terapia de Rerversão de Hábito; CDC = Grupo de

Decoupling.

${ }^{a}$ Um dos participantes abandonou o estudo durante o procedimento de intervenção;

b 03 participantes não realizaram a reavaliação;

${ }^{\mathrm{c}} 05$ participantes não realizaram a reavaliação; ${ }^{\mathrm{d}} \mathrm{GI}=$ pacientes que tinham acabado procurando serviço de dermatologia; ${ }^{\mathrm{e}} \mathrm{G} 2$ =pacientes que procuraram serviço dermatologia há aproximadamente 02 anos;

f02 participantes foram excluídos da análise por falta de dados.

Tabela 3 
Delineamento de pesquisa adotados nos estudos.

\begin{tabular}{|c|c|}
\hline $\begin{array}{l}\text { Delineamento pesquisa } \\
\text { utilizado }\end{array}$ & Artigos \\
\hline Sujeito único $\mathrm{AB}$ & $\begin{array}{l}\text { Capriotti et al (2015); Deckersbach et al. (2002) })^{\mathrm{a}} \text {; Martinson et al. (2011); } \\
\text { Rosenbaum \& Ayllon }(1981)^{\mathrm{a}} \text {; Twohig et al. }(2001)^{\mathrm{a}} \text {; Twohig et al. }(2006)^{\mathrm{a}}\end{array}$ \\
\hline Grupo sem controle & Flessner et al. (2007); Grillo et al. (2007) \\
\hline Grupo com controle & $\begin{array}{l}\text { Moritz et. al. (2012) })^{\mathrm{b}} \text {; O’Connor et al. (2018) }{ }^{\mathrm{a}} \text {; Shuck et al. (2011); Teng et al. } \\
(2006)^{\mathrm{a}}\end{array}$ \\
\hline $\begin{array}{l}\text { Linha de base múltipla e } \\
\text { linha de base com reversão }\end{array}$ & Flessner et al. (2008) \\
\hline Relatos de caso & $\begin{array}{l}\text { Fruensgaard (1991a); Ibiloglu et al. (2016); Jagger e Sterner (2016); Long et. al } \\
\text { (2006); Pearlstein e Orentreich (1968) }\end{array}$ \\
\hline
\end{tabular}

Instrumentos Utilizados nos Artigos.

\begin{tabular}{|c|c|}
\hline Artigos & Instrumentos \\
\hline Deckersbach et al. (2002) & $\begin{array}{l}\text { SPS (Skin Picking Scale); BDI-II (Beck Depression Inventory -II); Clinical Global } \\
\text { Improvement Scale; Structured Clinical Interview for DSM-IV Axis I Disorders }\end{array}$ \\
\hline Flessner et al. (2007) & Ficha de autorregistro; SPS (The Skin Picking Scale) \\
\hline Flessner et al. (2008) & Ficha de autorregistro; Massachusetts General Hospital Hairpulling Scale (MGH-HS) \\
\hline Rosenbaum e Ayllon (1981) & Registro em sessão \\
\hline Schuck et al. (2011) & $\begin{array}{l}\text { SPIS (Skin Picking Impact Scale); SPS (The Skin Picking Scale); SCCQ (Self-Control } \\
\text { Cognitive Questionnaire); Análise de fotografias; Ficha de autorregistro }\end{array}$ \\
\hline Teng et al. (2006) & $\begin{array}{l}\text { Ficha de autorregistro; Análise de fotografias; Treatment Evaluation Inventory-Short Form } \\
\text { (TEI-SF); Wechsler Abbreviated Scale of Intelligence (WASI) }\end{array}$ \\
\hline Twohig e Woods (2001) & Ficha de autorregistro; SITS (Self-Injury Trauma Scale); Análise de fotografias \\
\hline Twohig et al. (2006) & $\begin{array}{l}\text { Ficha de autorregistro; Objective Skin-damage ratings; SPIS (Skin Picking Impact Scale); } \\
\text { Massachusetts General Hospital Hairpulling Scale (MGH-HS); BDI-II (Beck Depression } \\
\text { Inventory); Beck Anxiety Inventory (BAI; Acceptance and Action Questionnaire (AAQ); } \\
\text { Treatment Evaluation Inventory-Short Form (TEI-SF) }\end{array}$ \\
\hline Capriotti et al. (2015) & $\begin{array}{l}\text { Ficha de autorregistro; SPS-R (Skin Picking Scale-Revised); MIDAS (Milwaukee Inventory for } \\
\text { the Dimensions of Adult Skin Picking) }\end{array}$ \\
\hline Martinson et al. (2011) & $\begin{array}{l}\text { BDI -II (Beck Depression Inventory - Second Edition); STAI (State/Trait Anxiety } \\
\text { Questionnaire); SPS (Skin Picking Scale); SPIS (Skin Picking Impact Scale); MIDAS } \\
\text { (Milwaukee Inventory for the Dimensions of Adult Skin Picking); Automonitoramento; Fotos }\end{array}$ \\
\hline Jagger e Sterner $(2016)^{a}$ & $\begin{array}{l}\text { Skin Picking Scale-Revised (SPS-R); SPIS (Skin Picking Impact Scale); MIDAS (Milwaukee } \\
\text { Inventory for the Dimensions of Adult Skin Picking) }\end{array}$ \\
\hline Ibiloglu et al. (2016) & Nenhum \\
\hline Moritz et al. (2011) & $\begin{array}{l}\text { Questionário de linha de base; M-SPS (Versão modificada da Skin Picking Scale); BDI - SF } \\
\text { (Beck Depression Inventory - Short Form) }\end{array}$ \\
\hline Pearlstein e Orentrich (1968) & Fotos \\
\hline O'Connor et al. (2018) & $\begin{array}{l}\text { BAI (Beck anxiety inventory); BDI- II (Beck Depression Inventory); MPS (Frost } \\
\text { multidimensional perfectionism scale); BIS -10 (Barratt impulsiveness scale); PI-R (Padua } \\
\text { Inventory-revised); SSEI (Social self-esteem inventory); STOP (Style of Planning } \\
\text { Questionnaire); Self-rated tension scale; Devilly-Borkovec credibility/expentacy } \\
\text { questionnaire; Nijimeng motivation list 2; Diário de autorregistro; MGH-HP (Massachusetts } \\
\text { general hospital hairpulling scale); TSGC-A (Versão adaptada -Tourette syndorme global } \\
\text { scale); Personality Diagnostic Questionnaire - Forth Edition; Reven's Matrice; Anxiety } \\
\text { Disordes Structures Interview Schedule for DSM-IV }\end{array}$ \\
\hline
\end{tabular}

${ }^{a}$ O estudo de Jagger e Sterner (2016) sugere esses instrumentos para avaliar, porém não realiza avaliação. 


\section{Procedimentos Adotados nas Intervenções}

Em relação aos procedimentos e estratégias adotados ao longo das intervenções, constatou-se que a Terapia de reversão de hábitos (TRH) foi adotada em dez dos 18 estudos. A TRH é uma técnica comportamental usada com o objetivo de diminuir a frequência de comportamentos repetitivos, que inclui cinco componentes a serem treinados: conscientização, relaxamento, resposta concorrente, apoio social e generalização (Azrin \& Nunn, 1973).

Dentre os estudos que adotaram a TRH, quatro utilizaram a estratégia como procedimento único de intervenção (Grillo et al. 2007; Rosenbaum e Ayllon, 1981; Teng et al., 2006; Twohig \& Woods, 2001) e seis utilizaram a TRH associada a outros procedimentos (Capriotti et al., 2015; Deckersbach et al., 2011; Flessner et al., 2008; Long et al., 2006; Jagger \& Sterner, 2016; Martinson et al., 2011), tais como estratégias de intervenção psicoeducacionais e a ACT. $\mathrm{O}$ procedimento que combinou TRH e a ACT foi denominado de Acceptance-Enhanced Behavior Therapy (AEBT). Um estudo comparou a TRH a outra intervenção chamada decoupling, que consiste em orientar o paciente a realizar um movimento com as mãos que se assemelha ao movimento realizado no transtorno de escoriação, porém direcionado a outro objetivo (e.g., encostar na orelha; Moritz et al., 2012).

Dois estudos utilizaram intervenções pontuais e parciais da TRH, apesar de não terem sido descritas dessa forma nos estudos. Perarlstein e Orentreich (1968) manejaram antecedentes ao utilizarem como procedimento um curativo como barreira física para inviabilizar a escoriação, condição que pode se relacionar à ocorrência de respostas concorrentes. Ibuloglu et al. (2016) propuseram a realização de uma técnica de imitação do comportamento de escoriar com função de eliciar sentimentos similares aos que ocorriam durante a escoriação e realizaram orientações para automonitoramento, sugerindo a utilização de um diário.

A TRH não foi utilizada como procedimento de intervenção comportamental em cinco estudos. Nesses, as intervenções foram conduzidas por meio da ACT (Twohig et al., 2006), intervenção cognitivocomportamental baseada em um manual elaborado para o estudo, que não foi descrito na publicação (Schuck et al., 2011) e, em uma proposta, intervenção psicoeducacional vinculada à internet (Flessner et al., 2007). O'Connor et al. (2018) propuseram o CoPs, um modelo de intervenção cognitiva fisiológica baseado em técnicas comportamentais que intervém nos pensamentos de impulsividade, perfeccionismo e planejamento. $\mathrm{O}$ autor descreveu que o tratamento inclui as seguintes fases: (a) descrição das situações de baixo e alto risco para ocorrência do comportamento de hábito; (b) autoconhecimento sobre as formas de interagir com essas situações; (c) autoconhecimento sobre estratégias que o cliente utiliza para interagir com as situações de baixo risco, de forma que estas possam auxiliar no manejo das de alto risco; (d) modificação das formas de agir diante das situações de alto e de baixo risco; (e) desenvolvimento de flexibilidade em relação ao perfeccionismo; e (f) desenvolvimento de plano de ação. O estudo de Fruensgaard (1991a) propôs que a intervenção deveria produzir (a) aliança terapêutica; (b) autoconhecimentos sobre os episódios de escoriação; (c) conhecimento sobre fatos da história de vida que pode se relacionar com as dificuldades atuais; (d) identificação dos conflitos que coincidiram com o início dos episódios de escoriação; (e) desenvolvimento de comportamentos mais funcionais e (f) apoio e orientação em relação as interações sociais.

Os componentes utilizados durante procedimentos de intervenção, em sua maioria, portanto, estão relacionados à TRH. Dentre esses estudos, dez realizaram treino de resposta concorrente (Capriotti et al., 2015; Deckersbach et al., 2002; Flessner et al., 2007, 2008; Grillo et al., 2007; Long et al.,2006; Martinson et al., 2011; Rosenbaum \& Ayllon, 1981; Teng et al., 2007; Twohig \& Woods, 2001). Em nove estudos foi realizado o treino de conscientização (Deckersbach et al., 2002; Flessner et al., 2007, 2008; Grillo et al., 2007; Long et al.,2006; Martinson et al., 2011; Rosenbaum \& Ayllon, 1981; Teng et al., 2007; Twohig \& Woods, 2001).

O componente de suporte social foi incluído em três estudos. Teng et al. (2007) solicitaram que o participante identificasse algum familiar próximo e capacitado para auxiliar na manutenção do tratamento. Twohig e Woods (2001), ao aplicar a intervenção, elogiaram os esforços do cliente, e Flessner et al. (2008) orientaram os participantes a pedir ajuda. A descrição de todas as intervenções relacionadas à terapia de reversão de hábito está detalhada na Tabela 5.

Dentre os procedimentos não relacionados à TRH destacam-se componentes presentes na ACT, tais como: distinguir entre o sentimento de necessidade do comportamento de escoriação e a prática do comportamento (Twohig et al., 2006); demostrar que eliminar as necessidades de escoriação não é efetivo e que emoções aversivas devem ser aceitas como naturais (i.e., as tentativas de controlar a escoriação podem ser o problema e não a solução; Capriotti et al., 2015; Twohig et al., 2006); mudar a função psicológica da necessidade de escoriação para evento verbal (defusion; Twohig et al., 2006); favorecer a identificação de valores e como o comportamento repetitivo afeta isso (Capriotti et al., 2015; Flessner et al., 2008), reconhecer as situações aversivas diante das quais engaja-se em controlar os impulsos e outras experiências negativas relacionadas ao comportamento repetitivo (Capriotti et al., 2015; Flessner et al., 2008; Twohig et al., 2006) e realizar atividades vivenciais (Capriotti et al., 2015; Twohig et al., 2006). 
Tabela 5.

Distribuição dos Artigos de Acordo com os Tipos de Procedimentos da Terapia de Reversão de Hábito Utilizados.

\begin{tabular}{|c|c|}
\hline Procedimentos & Intervenção terapêutica \\
\hline $\begin{array}{l}\text { Treino de } \\
\text { conscientização }\end{array}$ & $\begin{array}{l}\text { - Descrever antecedentes, respostas e consequências da escoriação (Flessner et al., 2007; Grillo et al., } \\
\text { 2007; Long et al., 2006; Martinson et al.2011Teng et al., 2007; Twohig \& Woods, 2001) } \\
\text { - Simular resposta de escoriação (Teng et al., 2007; Twohig, \& Woods, 2001) } \\
\text { - Autorregistro daescoriação (Capriotti et al., 2015; Deckersbach et al., 2002; Flessner et al., 2007, 2008; } \\
\text { Grillo et al., 2007; Long et al., 2006; Martinson et al., 2011; Rosenbaum \& Ayllon, 1981; Teng et al., } \\
\text { 2007; Twohig \& Woods, 2001) }\end{array}$ \\
\hline $\begin{array}{l}\text { Treino de Resposta } \\
\text { Concorrente }\end{array}$ & $\begin{array}{l}\text { - Estabelecer e ensinar respostas concorrentes à escoriação (Capriotti et al., 2015; Deckersbach et al., } \\
\text { 2002; Flessner et al., 2007, 2008; Grillo et al., 2007; Long et al., 2006; Martinson et al., 2011; } \\
\text { Rosenbaum \& Ayllon, 1981; Teng et al., 2007; Twohig \& Woods, 2001) } \\
\text { - Favorecer o treino da resposta concorrente à escoriação na sessão (Rosenbaum \& Ayllon, 1981; Twohig } \\
\text { \& Woods, 2001) } \\
\text { - Orientar a realização de atividades prazerosas (Flessner et al., 2008) } \\
\text { - Mudar postura e manter mãos afastadas da escoriação (Flessner et al., 2007) } \\
\text { - Utilizar objetos para manter as mãos ocupadas (Capriotti et al., 2015) }\end{array}$ \\
\hline $\begin{array}{l}\text { Treino de resposta } \\
\text { concorrente com uso de } \\
\text { controle de estímulos } \\
\text { antecedentes e } \\
\text { consequentes }\end{array}$ & $\begin{array}{l}\text { - Utilizar lembretes (Deckersbach et al., 2002; Capriotti et al., 2015) } \\
\text { - Usar barreiras físicas (e.g., luva, cachecol, band-aid, roupas) para afastar as mãos da pele (Deckersbach } \\
\text { et al., 2002; Flessner et al., 2007, 2008; Martinson et al., 2011; Pearlstein \& Orentrich, 1968; Schuck } \\
\text { et al., 2011) } \\
\text { - Trocar de roupa na frente de outras pessoas (Deckersbach et al., 2002) } \\
\text { - Consultar profissional de saúde (Flessner et al. 2007) } \\
\text { - Evitar, cobrir espelhos e diminuir a luz (Capriotti et al., 2015; Flessner et al., 2007) } \\
\text { - Desfazer-se de instrumentos de escoriação (Flessner et al., 2007; Schucket al., 2011) } \\
\text { - Fazer as unhas (Flessner et al., 2007) } \\
\text { - Usar pomadas para evitar a escoriação (Flessner et al., 2007) } \\
\text { - Evitar situações antecedentes à escoriação (Flessner et al., 2007) } \\
\text { - Deixar luz acessa na frente de outros (Martinson et al., 2011) } \\
\text { - Tirar maquiagem na pia da cozinha (Capriotti et al., 2015) } \\
\text { - Instruir o engajamento em atividades reforçadoras (e.g., evento social, comprar presente) após um } \\
\text { período sem escoriação (Deckersbach et al., 2002) }\end{array}$ \\
\hline Treino de relaxamento & - Ensinar estratégias de relaxamento (Flessner et al., 2007; Martinson et al., 2011) \\
\hline $\begin{array}{l}\text { Treino de Suporte } \\
\text { Social }\end{array}$ & $\begin{array}{l}\text { - Identificar pessoas para dar suporte social e elogiar quando a resposta concorrente for usada } \\
\text { adequadamente (Teng et al., 2007) } \\
\text { - Instruir para o treinamento de pessoas significativas para dar suporte social e elogiar respostas } \\
\text { concorrentes (Teng et al., 2007) } \\
\text { - Elogiar os esforços do cliente (Twohig \& Woods, 2001) } \\
\text { - Orientar a pedir ajuda (Flessner et al., 2008) }\end{array}$ \\
\hline $\begin{array}{l}\text { Treino de } \\
\text { generalização }\end{array}$ & -Instruir que as respostas concorrentes sejam realizadas em diversos ambientes (Martinson et al., 2011) \\
\hline
\end{tabular}

\section{Análise qualitativa dos resultados dos estudos}

Todas as intervenções apresentadas nos estudos mostraram maior ou menor grau de efetividade, uma vez que foi constatada redução na frequência de escoriação, mas que variou de um estudo para outro. No estudo de Twohig e Woods (2001) a frequência do comportamento de escoriação reduziu após a intervenção. Entretanto, na sessão de avaliação de seguimento para os dois participantes, a frequência aumentou em nível inferior à pré-intervenção. Quanto à avaliação de fotografias das áreas lesionadas da pele, os autores afirmaram que as fotos das áreas com escoriações na fase pós-intervenção foram melhor avaliadas do que aquelas tiradas das mesmas áreas na pré-intervenção. Dados semelhantes foram obtidos por meio da escala SITS (Self Injury Trauma Scale; Iwata, Pace, Kissel, Nau, \& Farber, 1990).

Em concordância com os resultados obtidos por Twohig e Woods (2001), Deckersbach et al. (2002) descreveram três estudos de caso. Como procedimento de intervenção nos três casos, aplicou-se a terapia cognitivocomportamental, que não teve sua forma de aplicação descrita no estudo, combinada com a reversão de hábito para pacientes com escoriação e comorbidades psiquiátricas. Ao final da intervenção, constatou-se melhora na condição dos comportamentos de escoriação, por meio da redução dos scores das escalas SPS (The Skin Pikcing Scale; Keuthen et al., 2001a) e BDI II (Beck Depression Inventory; Beck, Steer, \& Brown, 1996) nas 
etapas pós-intervenção. Esta condição foi mantida na avaliação de seguimento.

O estudo de Teng et al. (2007) utilizou o delineamento experimental entre grupos. Verificou-se que os indivíduos do grupo que recebeu intervenção, por meio da terapia de reversão de hábito (THR), apresentaram redução significativa do comportamento de escoriação, quando comparado ao grupo controle. A manutenção dos resultados pode ser observada nas sessões de avaliação de seguimento. Embora não tenha havido redução dos comportamentos de escoriação entre os participantes do grupo controle, observou-se diminuição da frequência relatada. Tal diminuição parece não corresponder aos dados da análise das fotos, nas quais não se contatou diferença entre as fases de pré e pós-tratamento, bem como na avaliação de seguimento. Moritz et al. (2012) utilizaram o delineamento de grupo para comparar o tratamento autoaplicável de TRH e decoupling e concluíram que a diminuição dos sintomas foi de $50 \%$ no grupo de TRH e de $33 \%$ no outro grupo.

Flessner et al. (2008) utilizaram a AcceptanceEnhanced Behavior Therapy (AEBT), que consiste em procedimentos de reversão de hábito combinados com ACT, no tratamento de escoriação e tricotilomania. Dentre os principais resultados do estudo, se evidenciou melhoras visuais da pele e diminuição da frequência do comportamento, segundo relato dos participantes. Além disso, constatou-se que a ordem de apresentação dos procedimentos de ACT e reversão de hábito não influenciou na efetividade da intervenção. Capriotti et al. (2015) também utilizaram a AEBT em quatro intervenções individuais e concluíram que todos os participantes obtiveram diminuição dos escores nas escalas SPS-R (Skin Picking Scale-Revised; Snorrasson et al., 2012) e MIDAS (Miwaulkee Inventory for Dimesions for Adult Skin Picking; Walther, Flessner, Conelea, \& Woods, 2009) e diminuição do tempo de escoriação por dia, conforme dados de autorrelato.

Outro estudo que utilizou estratégias baseadas na ACT foi o de Twohig et al. (2006). Após a intervenção, quatro dos cinco participantes relataram, por meio do autorregistro, redução na frequência do comportamento de escoriação. Tal redução foi mantida apenas por um dos participantes durante a sessão de follow-up realizada após três meses da intervenção. Tais resultados também puderam ser observados nas escalas SPIS (Skin Picking Impact Scale; Keuthen et al., 2001b) e na análise das fotografias das áreas do corpo em que ocorria a escoriação. Houve também redução da frequência do comportamento autolesivo na pele, após a intervenção psicoeducacional conduzida por meio de uma plataforma online (Flessner et al., 2007). Como resultado do estudo, observou-se que os participantes que utilizaram a plataforma por períodos maiores de tempo tiveram reduções mais significativas do comportamento de escoriação do que indivíduos que utilizaram a plataforma por períodos menores.

O'Connor et al. (2018) ao aplicar CoPs concluíram que houve diminuição significativa de escores nas fases de pré- e pós-tratamento nas escalas TSGS-A
(Tourette Syndrome Global Scale - Adapted Version). BAI (Beck Depression Inventory) e BDI-II (Beck Anxiety Inventory). Na avaliação de seguimento seis meses após o tratamento, os resultados foram mantidos. O'Connor et al. (2018) concluíram que dos participantes que participaram da intervenção, $74 \%$ obtiveram melhoras significativas e $4 \%$ mostraram piora. No grupo controle, a porcentagem de melhora foi $33 \%$ e de piora, 25\% (O'Connor et al., 2018).

Os estudos de caso também indicaram intervenções bem-sucedidas. Pearlstein e Orentrich (1968), que utilizaram um curativo costurado como barreira para evitar o contato do paciente com o local de ferida, descreveram cicatrização da pele como principal resultado. Ibiloglu et al. (2016), ao orientarem o paciente sobre as estratégias de imitação do comportamento de escoriação e automonitoramento, obtiveram como resultado a redução de ansiedade, frequência e intensidade da escoriação.

\section{DISCUSSÃO}

A partir da revisão bibliográfica realizada, aspectos relacionados à condição atual das pesquisas e aplicações sobre transtorno de escoriação devem ser discutidos. A primeira pesquisa aplicada encontrada nesse estudo sobre o tema foi realizada por Perarlstein e Orentreich (1968), sendo que desde essa data até 2000, apenas outras duas foram publicadas (Fruesngaard, 1991a; Rosenbaum \& Ayllon, 1981). A partir do ano 2000, ocorreu um aumento no interesse dos pesquisadores pelo tema. Isso pode ter contribuído para a melhor delimitação da condição, que só passou a apresentar uma classificação específica no DSM V, em 2013 (APA, 2013).

Após essa análise das pesquisas sobre o tratamento do transtorno de escoriação, discussões devem ser realizadas sobre os métodos adotados, as intervenções realizadas e a generalização dos resultados. Em relação ao método empregado nos estudos, observa-se que existem muitas semelhanças entre os métodos, especialmente em relação aos participantes e os indicadores utilizados. Os participantes eram adultos com idade superior a 18 anos. Essa é uma condição interessante, uma vez que se houvesse muita diversidade em relação a isto, possíveis diferenças de resultados poderiam ser atribuídas a essa variável. Entretanto, as pesquisas sobre escoriação com crianças e adolescentes são escassas, sendo necessário aperfeiçoar os estudos, especialmente com esta população. É provável que existam especificidades em relação ao procedimento para essa população e isto poderia ser alvo de estudos, de modo a proporcionar intervenções mais efetivas. Dentre as principais diferenças em relação aos procedimentos direcionados a esta população, destaca-se a participação ou a mediação de pais e/ou responsáveis. Existem, de modo geral, poucos estudos que abordem aspectos psicológicos e comportamentais em pacientes de dermatologia pediátrica (Perry \& Streusand, 2013).

Como os indicadores da resposta de escoriação foram obtidas por meios indiretos, pode-se discutir ainda sua precisão. Dentre os estudos revisados, nenhum avaliou o comportamento de escoriação de modo direto, por meio de filmagens e categorização da taxa de respostas. Parece 
haver, portanto, uma impossibilidade de se avaliar diretamente o comportamento, devido à maior probabilidade de emissão destes em situações privadas (Zazula, Sartor, Dias, \& Gon, 2014), nas quais o pesquisador ou o profissional de saúde não tem acesso direto. Um dos indicadores empregados foi a utilização de fichas de autorregistro, que possuem dupla função: autoobservação para o cliente e obtenção de dados para a realização de uma análise funcional do comportamento de escoriação. Mesmo que em pesquisas futuras surjam novas estratégias para mensurar a frequência do comportamento de escoriação, o autorregistro continuará sendo importante enquanto indicador e estratégia para a avaliação e intervenção, devido à sua função de auto-observação para o paciente.

Ao se avaliar as intervenções realizadas, pode-se afirmar que os componentes da TRH foram significativamente utilizados. Além disso, não foi possível constatar, dentre os estudos selecionados, delimitação clara sobre o número de sessões necessárias para a efetividade do tratamento. Todos os estudos analisados foram efetivos no tratamento do transtorno de escoriação, independentemente do número de sessões conduzidas com os participantes, conforme descrito na seção de resultados. Neste sentido, a delimitação da quantidade de sessões parece não ser a variável significativa para avaliar a efetividade ou não do tratamento.

Em relação à efetividade da intervenção, observase que alguns estudos constataram que as intervenções foram efetivas para a redução da frequência de comportamento de escoriação. Entretanto, a manutenção dos resultados não é clara dentre os estudos disponíveis sobre o tema e selecionados para o presente estudo. Isso pode estar relacionado ao fato das intervenções serem técnicas e terem sido conduzidas sem uma delimitação clara da funcionalidade dos comportamentos emitidos e definidos como escoriação, especialmente quando realizadas em delineamento de grupo. Selles et al. (2016) afirmam que estudos sobre a formas de aplicação da terapia comportamental para o tratamento do transtorno de escoriação são necessários para a compreensão da efetividade da mesma. Neste sentido, como sugestão de pesquisas futuras, indica-se a realização de estudos de intervenção com participantes de diferentes faixas etárias (i.e., especialmente crianças e adolescentes) e, de pesquisas com delimitação e descrição funcional dos comportamentos de escoriação. A aplicação da TRH com maior frequência, de modo a identificar com clareza as variáveis responsáveis pela efetividade desta estratégia de intervenção nos casos de transtorno de escoriação pode favorecer a avaliação da função deste comportamento. A investigação de variáveis, a partir dos métodos e das técnicas da ciência, auxilia na previsão e controle do comportamento (Skinner, 1953/2003).

Neste sentido, os princípios envolvidos na aquisição, manutenção e possível alteração do comportamento de escoriação são importantes. É relevante a realização da análise funcional para a compreensão das variáveis envolvidas na emissão deste comportamento, bem como todo o processo comportamental de alteração do mesmo. De acordo com Gôngora (2003), compreender e intervir apenas na topografia dos comportamentos ajudam muito pouco na compreensão do comportamento como um todo. Talvez esta seja uma das razões que levaram inúmeras intervenções direcionadas a este problema, especialmente durante a avaliação de seguimento, não manterem seu resultado inicial de redução de frequência. Se o foco estiver em modificar o comportamento do ponto de vista topográfico, e não em compreender sua função no repertório comportamental do indivíduo que o emite, faltarão dados para uma aplicação eficaz dos procedimentos.

\section{REFERÊNCIAS}

American Psychiatric Association. (2000). Diagnostic and Statistical Manual of Mental Disorders (4th ed. Rev.). Washington, DC: Author.

American Psychiatric Association. (2013). Diagnostic and Statistical Manual of Mental Disorders (5th ed.). Washington, DC: Author.

Azrin, N.H., \& Nunn, R.G. (1973). Habit-reversal: A method of eliminating nervous habits and tics. Behaviour Research and Therapy, 11(4), 619-628. doi:10.1016/0005-7967(73)90119-8

Beck, A.T., Steer, R.A., \& Brown, G.K. (1996). Beck Depression Inventory-II. San Antonio, TX: The Psychological Corporation.

Capriotti, M.R., Ely, L.J., Snorrason, I., \& Woods, D.W. (2015). Acceptance-enhanced bahevior therapy for excoriation (skin picking) disordes in adultd: A clinical case series. Cognitive and Behavioral Parctice, 22, 230-239. doi: 10.1016/jcbpra.2014.008

Deckersbach, T., Wilhelm, S., Keuthen, N.J., Baer, L., \& Jenike, M.A. (2002). Cognitive-behavior therapy for self-injurious skin picking. A case series. Behavior Modification, 26(3), 361-377. Recuperado de http://www.ncbi.nlm.nih.gov/pubmed/12080906

Flessner, C.A., Moutoun-Odum, S., Stocker, A.J., \& Keuthen, N.J (2007). StopPicking.com: Internet-based treatment for self-injurius skin picking. Dermatology Online Journal, 13(4), 3. Recuperado de https://escholarship.org/uc/item/1dx97474

Flessner, C.A., Busch, A.M., Heideman, P.W., \& Woods, D.W. (2008). Acceptance-enhanced behavior therapy (AEBT) for trichotillomania and chronic skin picking: exploring the effects of component sequencing. Behavior Modification, 32(5), 579-594. doi:10.1177/0145445507313800

Fruensgaard, K. (1991a). Phychotherapeutic strategy and neurotic excoriations. International Journal of Dermatology, 30 (3), 198-203. doi: 10.1111/j.13654362.1991.tb03851.x

Fruensgaard, K. (1991b). Phychothery and neurotic excoriations. International Journal of Dermatology, 30 (3), 262-265. doi: 10.1111/j.13654362.1991.tb04634.x

Gon, M.C.C., Gon, A.S., \& Zazula, R. (2013). Análise comportamental de relatos verbais de mães de crianças com dermatose crônica. Revista Brasileira de Terapia Comportamental e Cognitiva, 15, 57-71. 
Gon, M.C.C., Rocha, M.M., \& Gon, A.S. (2005). Análise do conceito de estigma em crianças com dermatoses crônicas. Revista Brasileira de Terapia Comportamental e Cognitiva, 7, 15-20.

Gôngora, M.A.N. (2003). Noção de psicopatologia na Análise do Comportamento. In: C.E. Costa, J.C. Luzia, \& H.H.N. Sant'Ana. Primeiros passos na Análise do Comportamento e Cognição (pp. 93-109). Santo André: ESETEC.

Grant, J.E., \& Odlaug, B.L. (2009). Update on pathological skin picking. Current Psychiatry Reports, 11, 283-288. doi:10.1007/s11920-009-0041-x

Grillo, M., Long, M., \& Long, D. (2007). Habit reversal training for the itch-scratch cycle associated with pruritic skin conditions. Dermatology Nursing, 19, 243-248.

Gupta, M., \& Gupta, A.K. (1996). Psychodermatology: An update. Journal of the American Academy of Dermatology, 34, 1030-1046. doi: 10.1016/S01909622(96)90284-4

Harth, W., Taube, K.M., \& Gieler, U. (2010). Facticious disorders in dermatology. Journal Der Deutschen Dermatologischen Gesellschaft [Journal of the German Society of Dermatology], 8(5), 361-372. doi:10.1111/j.1610-0387.2010.07327.x

Ibiloglu, O.A., Atli, A., Kaya, M.C., Demir, S., Bulut, M., $\&$ Sir, A. (2016). A Case of Skin Picking Disorder of a patient with a history of childhood abuse.Archives of Neurophychiatric, 53, 181-183. doi: 10.5152/npa.201510110

Iwata, B.A., Pace, G.M., Kissel, R.C., Nau, P.A, \& Farber, J.M. (1990). The Self-Injury Trauma (SIT) Scale: A method for quantifying surface tissue damage caused by self-injurious behavior. Journal of Applied Behavior Analysis, 23, 99-110. https://doi.org/10.1901/jaba.1990.23-99

Jagger, G.E, \& Sterner, W.R. (2016). Excoriation: What counselors need to know about skin picking disorder. Journal of Mental Health Counseling, 38 (4), 281-297. https://doi.org/10.17744/mehc.38.4.01

Keuthen, N.J., Wilhelm, S., Deckersbach, T., Engelhard, I., Forker, A.E., Baer, L, \& Jenike, M.A. (2001a). The Skin Picking Scale: Scale construction and psychometric analyses. Journal of Psychosomatic Research, 50, 337-341. https://doi.org/10.1016/S00223999(01)00215-X

Keuthen, N.J., Deckerbach, T., Wilhem, S., Engelhard, I., Forker, A.E., O' Sullivan, R.L., Jenike, M.A., \& Baer, L. (2001b). The Skin Picking Impact Scale (SPIS): Scale development and psychometric analyses. Psychosomatics, 42, 397-403.

Keuthen, N.J., Tung, E.S., Reese, H.E., Raikes, J., Lee, L., \& Mansueto, C.S. (2015). Getting the word out: Cognitive-bahavioral therapy for trichotillomania (hair-pulling disorder) and excoriation (skin-picking) disorder. Annals of Clinical Psychiatry, 27 (1), 10-15.

Lochner, C., Roos, A., \& Stein, D.J. (2017). Excoriation (skin-picking) disorder: A systematic review of treatment options. Neuropsychiatry Disease and
Treatment, 14, 1867-1872. doi: 10.2147/NDT.S121138. eCollection 2017.

Long, D., Long, R., Grillo, M., \& Marshman, G. (2006). Development of a pshychological treatment service for prurutic skin conditions. Australasian Journal of Dermatology, 47(4), 237-241. doi:10.1111/j.14400960.2006.00288.x

Martinson, A.A., Nangle, D.W., Boulard, N., \& Sigmon, S. (2011). Old habits die hard: Treating a woman with a 20-year severe case of skin picking disorder. Clinical Case Studies, 10, 411-426. doi: 10.1177/1534650111428508.

Moritz, S., Fricke, S., Treszl, A., \& Wittekind, C.E.(2012). Do it yourself! Evaluation of self-help habit reversal training versus decoupling in pathological skin picking: A pilot case. Journal of ObsessiveCompulsive and Related Disorders, 1, 41-47. doi: 10.1016/j.jocrd.2011.11.001

Miltenberger, R.G., Fuqua, R.W., \& Woods, D.W. (1998). Applying behavior analysis to clinical problems: Review and analysis of habit reversal. Journal of Applied Behavior Analysis, 31, 447-469. doi:10.1901/jaba.1998.31-447

O'Connor, K., Lavoie, M., Desaulniers, B., \& Audet, J.S. (2018). Cognitive psychophysiological treatment of bodily-focused repetitive behaviors in adults: An open trial. Journal of Clinical Psychology, 74, 273-285. doi: 10.1002/jclp.22501. Epub 2017 Aug 16

Odlaug, B.L., \& Grant, J.E. (2010). Pathologic Escoriação. The American Journal of Drug and Alcohol Abuse, 36, 296-303. doi:0.3109/00952991003747543

Pearlstein, H.H., \& Orentreich, N. (1968). Sutured Dressing: An Adjunct in the Treatment of Neurotic Excoriations. Archives of Dermatology, 98(5), 508511. doi:10.1001/archderm.98.5.508

Perry, M., \& Streusand, W.C. (2013). The role of psychiatry and psychology collaboration in pediatric dermatology. Dermatologic Clinics, 31, 347-355. https:// 10.1016/j.det.2012.12.012

Rosenbaum, M.S., \& Ayllon, T. (1981). The behavioral treatment of neurodermatitis through habit-reversal. Behaviour Research and Therapy, 19(4), 313-318. doi:10.1016/0005-7967(81)90052-8

Shah, K.N., \& Fried, R.G. (2006). Factial dermatoses in children. Current Opinion in Pediatrics, 18, 403-409. doi: 10.1097/01.mop.0000236390.41462.39

Schuck, K., Keijsers, G.P., \& Rinck, M. (2011). The effects of brief cognitive-behaviour therapy for pathological skin picking: A randomized comparison to wait-list control. Behaviour research and therapy, 49(1), 11-17.doi:10.1016/j.brat.2010.09.005

Selles, R.J, McGuire, J. F., Small, B. J., \& Storch, E. A (2016). A systematic review and meta-analysis of psychiatric treatments for excoriation (skin-picking) disorder. General Hospital Psychiatry, 41, 29-36. doi: dx.doi.org/10.1016/j.genhosppsych.2016.04.001

Skinner, B. (1953/2003). Ciência e comportamento humano. São Paulo: Martins Fontes.

Snorrason, I., Ólafsson, R.P., Flessner, C.A., Keuthen, N.J., Franklin, M.E., \& Woods, D.W. (2012). The Skin 
Picking Scale-Revised: Factor structure and psychometric properties. Journal of ObsessiveCompulsive and Related Disorders, 1, 133-137. https://doi.org/10.1016/j.jocrd.2012.03.001

Schumer, M.C, Bartley, C. A., \& Bloch, M.H (2016). Systematic review of pharmacological and behavioral treatments for skin picking disorders. Journal of Clinical Psychopharmacology, 36 (2), 147-152. doi:10.1097/JCP.0000000000000462

Teng, E.J., Woods, D.W., \& Twohig, M.P. (2006). Habit reversal as a treatment for chronic skin picking: A pilot investigation. Behavior Modification, 30(4), 411-422. doi:0.1177/0145445504265707

Twohig, M.P., Hayes, S.C., \& Masuda, A. (2006). A preliminary investigation of Acceptance and Commitment Therapy as a treatment for chronic skin picking. Behaviour Research and Therapy, 44(10), 1513-1522. doi:10.1016/j.brat.2005.10.002

Twohig, M.P., \& Woods, D.W. (2001). Habit reversal as a treatment for chronic skin picking in typically developing adult male siblings. Journal of Applied Behavior Analysis, 34(2), 217-220. doi:0.1901/jaba.2001.34-217

Ullman, L.P., \& Krasner, L. (1975). Psychological approach to abnormal behavior (2th ed.), Prentice Hall: New Jersey.

Walther, M.R., Flessner, C.A., Conelea, C.A., \& Woods, D.W. (2009). The Milwaukee Inventory for the Dimensions of Adult Skin Picking (MIDAS): Initial development and psychometric properties. Journal of Behavior Therapy and Experimental Psychiatry, 40, 127-135. https://doi.org/10.1016/j.jbtep.2008.07.002

Wetterneck, C.T., Teng, E.J., \& Stanley, M.A. (2010). Current issues in the treatment of OC-spectrum conditions. Bulletin of the Menninger Clinic, 74(2), 141-166.

Woods, D.W., \& Miltenberger, R.G. (1995). Habit reversal: A review of applications and variations. Journal of Behavior Therapy and Experimental Psychiatry, 26(2), 123-131. doi:10.1016/00057916(95)00009-O

Zazula, R., Sartor, M.S., Dias, N.G., \& Gon, M.C.C. (2014). Uso de medidas diretas e indiretas para avaliação de problemas de comportamento em crianças com dermatite atópica. In. V.B. Haydu, S.A. Fornazari, \& C.R. Estanislau (Orgs.). Psicologia e análise do comportamento: conceituações e aplicações à educação, organizações, saúde e clínica (pp. 267-287). Londrina: UEL. 